\title{
Manejo racional eleva o bem-estar de bovinos Guzerá e melhora a eficiência do trabalho de vacinação
}

Rational management raises the Guzerat cattle welfare and improves the efficiency of vaccination work

Marcos Chiquitelli Neto - Cristiane Gonçalves Titto •

José Nicolau Próspero Puoli Filho - Ana Luisa Silva Longo •

Thays Mayra da Cunha Leme-dos-Santos • Evaldo Antonio Lencioni Titto •

Leandro Zuccherato Camerro - Alfredo Manuel Franco Pereira

M Chiquitelli Neto - LZ Camerro

Departamento de Biologia e Zootecnia, Faculdade de Engenharia de Ilha Solteira, Universidade Estadual Paulista (UNESP), Av. Brasil 56, Ilha Solteira, SP 15385-000, Brasil.

CG Titto (Autor para correspondência) - ALS Longo TMC Leme-dos-Santos • EAL Titto

Universidade de São Paulo (USP), Faculdade de Zootecnia e Engenharia de Alimentos, Laboratório de Biometeorologia e Etologia, Departamento de Zootecnia.

email: crisgtitto@usp.br

\section{JNP Puoli Filho}

Faculdade de Medicina Veterinária e Zootecnia, Universidade Estadual Paulista Júlio de Mesquita Filho, Distrito de Rubião Junior, s/n Caixa Postal 560 Botucatu, SP 18618-970. Brasil.

\section{AMF Pereira}

Laboratório de Biometeorologia e Bem-Estar Animal, Instituto de Ciências Agrárias e Ambientais Mediterrânicas, Universidade de Évora, Apartado 94, Évora, 7006-554. Portugal.

Recebido: 06 de Julho, 2015 - Revisado: 29 de Setembro, 2015 • Aceito: 01 de Outubro, 2015

Resumo O experimento foi conduzido na seção de produção animal da Fazenda de Ensino, Pesquisa e Extensão (FEPE UNESP/Ilha Solteira). Foram utilizados 120 bovinos da raça Guzerá de diferentes idades, sendo: 40 fêmeas adultas, 40 machos jovens e 40 bezerros, dos quais 20 de cada categoria eram vacinados pelo método racional e os outros 20 pelo método convencional. Dentre os dados analisados, em relação à categoria de bezerros os indicadores de eficiência de manejo apresentaram médias menores quando submetidos ao manejo racional durante o processo de vacinação, com diminuição do tempo de execução do trabalho $(\mathrm{P}<0,001)$, introdução repetitiva da agulha e sangramento no local da aplicação $(\mathrm{P}<0,05)$; assim como nos percentuais de comportamentos como reagir à introdução da agulha $(\mathrm{P}<0,01)$, deslocamento $(\mathrm{P}<0,001)$, subir sobre outro animal e tentar fugir/pular do brete $(\mathrm{P}<0,05)$.Na categoria de fêmeas adultas, o manejo aplicado de forma racional também resultou em menores valores referentes aos indicadores de perda de dose de vacina $(\mathrm{P}<0,05)$, sangramento no local da aplicação, tempo de execução do trabalho e aplicação da vacina em local incorreto $(\mathrm{P}<0,001)$, bem como nos comportamentos de reagir à introdução da agulha $(\mathrm{P}<0,01)$ e deslocamento $(\mathrm{P}<0,001)$. Da mesma forma, os resultados referentes à categoria de machos jovens também demonstraram diferenças significativas em relação aos dois tipos de manejo empregados, observando-se no manejo racional menores médias em indicadores como tempo de execução do trabalho e aplicação da vacina em local incorreto $(\mathrm{P}<0,001)$, além de comportamentos como deslocamento $(\mathrm{P}<0,01)$ e tentativa de fugir/pular do brete $(\mathrm{P}<0,05)$.Neste contexto, nota-se que o manejo convencional demonstrou causar uma possível sensação de ameaça nos animais, ressaltando reações de fuga e medo que dificultam a rotina de manejo, causando perdas de material e de qualidade no produto final, além de aumentar os riscos de acidentes de trabalho devido a um comportamento mais agressivo em relação ao operador.

Palavras-chave: bovinocultura de corte, comportamento aversivo, curral, estresse 
Abstract The experiment was done in the Teaching, Research and Extension Farm (FEPE - UNESP/Ilha Solteira). One hundred and twenty Guzerat cattle of different ages were used in the study: 40 mature cows, 40 yearling steers and 40 calves. Twenty animals of each category were vaccinated by rational management and the other 20 by conventional method. For calves, when they were submitted to rational management during the vaccination process, the management efficiency indicators showed lower averages, with lower time of work execution ( $\mathrm{P}<0.001)$, repetitive introduction of the needle and bleeding at the injection site $(\mathrm{P}$ $<0.05)$; as well as on the behaviors like reacting to the introduction of needle $(\mathrm{P}<0.01)$, body movement $(\mathrm{P}<0.001)$, jump up on another animal and attempting to or/jumping out of chute $(\mathrm{P}<0.05)$. In the category of mature cows, the rational management also resulted in lower values of vaccine losses ( $\mathrm{P}<0.05)$, bleeding at the injection site, time of work execution and application of the vaccine in the wrong

\section{Introdução}

A procura por melhores índices produtivos, junto ao atendimento das exigências dos consumidores e de pressões exercidas para a implantação de meios que visem o bemestar, ressaltam a importância do manejo racional na propriedade, o qual funciona como uma ferramenta de melhoria na relação equipe de trabalho/animal, diminuindo riscos de acidentes e possíveis lesões na carcaça oriundas do uso de equipamentos e/ou de quedas e pisoteamento durante a condução.

O manejo realizado sem considerar as necessidades básicas dos animais causa um estresse excessivo, exercendo efeito negativo na produtividade e qualidade dos alimentos, podendo ocorrer quedas na produção, falhas na reprodução e crescimento, além de uma maior incidência de doenças e produção de carne de qualidade inferior (Pinheiro e Brito 2009). Durante muito tempo, a crescente preocupação somente com a obtenção de lucros fez com que uma visão mecanicista se sobressaísse entre os criadores. No entanto, este antigo entendimento de animais como "máquinas de produzir" perde força no cenário atual da pecuária de corte brasileira, estimulando o desenvolvimento de pesquisas que visem uma melhor compreensão das relações entre humanos e animais de produção.

Entretanto, antes de avaliar somente as condições físicas dos bovinos, devem ser analisadas as condições socioeconômicas das pessoas envolvidas no trabalho, baseando-se no fato de que boa parte ainda está submetida a condições precárias de sobrevivência, além de horas excessivas de trabalho. Apesar de não reconhecido, o trabalho do "peão" é de extrema importância na criação. A relação existente entre esse profissional e os animais influencia diretamente na execução das atividades, pois é location ( $\mathrm{P}<0.001)$, as well as some behaviors like reacting to the introduction of needle $(\mathrm{P}<0.01)$ and body movement ( $\mathrm{P}<0.001)$. Similarly, the results obtained for yearling steers also showed significant differences between the two types of management used, with lowest average in the rational management on the indicators such as time of work execution and application of the vaccine in the wrong location ( $\mathrm{P}<0.001)$, and behaviors such as body movement $(\mathrm{P}<0.01)$ and attempting to escape/jumping out of the chute ( $\mathrm{P}<0.05)$. In this context, it is noted that the conventional management of vaccination possibly caused a sense of threat in animals, emphasizing fear and escape reactions, making the routine management more difficult, causing loss material and quality loss in the final product (beef), and increase the risk of accidents due to more aggressive behaviour toward the operator.

Keywords: beef cattle, aversive behavior, corral, stress

durante o manejo onde ocorre uma maior interação, e o entendimento do animal por parte do homem possibilita evitar reações adversas.

Diversos estudos apontam os bovinos como animais adaptados a rotinas estabelecidas e providos de ótima memória, sendo capazes de discriminar pessoas envolvidas nas atividades, apresentando reações específicas a cada uma delas em função do tipo de experiência vivida. No caso de ações humanas aversivas, há uma tendência de aumento no nível de medo dos animais e, consequentemente, isso faz com que os mesmos se mostrem mais reativos quando submetidos à aproximação (Pajor, Rushen e De Passiélle 2000).

Neste contexto, o treinamento da equipe tem como objetivo orientar sobre novos métodos de manejo, visto que a maioria dos envolvidos, apesar de possuírem experiência prática notável na criação, muitas vezes possuem baixa escolaridade, tornando a aplicação de novos conhecimentos um desafio, ressaltando a necessidade do desenvolvimento de pesquisas que possam fundar uma base sólida sobre o assunto, indicando que novas estratégias podem, de fato, trazer ganhos diretos e/ou indiretos para todos os segmentos envolvidos com a produção de carne.

O trabalho teve como objetivo avaliar a influência de dois diferentes tipos de manejo sobre o bem-estar e a eficiência de trabalho do operador durante a vacinação de bovinos da raça Guzerá de diferentes idades.

\section{Material e Métodos}

O experimento foi conduzido no curral de manejo de bovinos na Seção de Produção Animal da Fazenda de Ensino, Pesquisa e Extensão da UNESP de Ilha Solteira (FEPE - UNESP/Ilha Solteira), aprovado pela Comissão de 
Ética no Uso de Animais (CEUA/FZEA/USP $\mathrm{n}^{\circ}$ 4069210915). As colheitas de dados foram realizadas no próprio curral de manejo, sendo este composto por mangas de apartação, seringa, brete coletivo e tronco de contenção. Os dados foram colhidos em três momentos distintos, conforme o programa de vacinação da fazenda. Em cada período foram utilizados 120 bovinos não mochados da raça Guzerá de diferentes idades, sendo: 40 fêmeas adultas (maior que 36 meses), 40 machos jovens (entre 18 e 24 meses) e 40 bezerros (entre 6 e 17 meses). Os tratamentos empregados no estudo foram relativos ao conjunto de ações realizadas durante o processo de vacinação, sendo: Tratamento RAC (Manejo Racional) e Tratamento CON (Manejo Convencional), onde 20 animais de cada categoria eram vacinados pelo método Racional e os outros 20 pelo método Convencional.

No tratamento racional, a colheita iniciava-se com o pente traseiro do brete aberto. Os animais eram conduzidos ao tronco de contenção, um a um, sem promover impacto na paleta ou pescoço, contidos apenas com a utilização da pescoceira. A vacina era aplicada no terço médio do pescoço com a pistola aplicadora na posição paralela ao corpo do animal. Durante o manejo não foi permitido o trânsito de pessoas na plataforma do brete, e o ferrão elétrico somente foi utilizado em casos de empacamento.

No tratamento convencional, a colheita de dados e o manejo de vacinação eram iniciados com o pente traseiro do brete coletivo aberto e com todos os animais preenchendo esse compartimento. Após a aplicação da vacina em todos os indivíduos, o pente dianteiro era aberto e os animais deixavam o local, um a um, em direção ao apartador. Após a saída do último animal era reiniciado o processo de preenchimento desse compartimento e, neste caso, não foi proibida a utilização de quaisquer instrumentos como ferrões elétricos e varas. Foram registrados dados de reatividade do animal ao manejo (tabela 1) e ações humanas durante o processo de aplicação da vacina, bem como as interações que ocorrem entre os animais e os operadores (tabela 2).

Tabela 1 Variáveis comportamentais observadas durante o programa de vacinação de bovinos em curral de manejo.

\begin{tabular}{|c|c|}
\hline $\begin{array}{l}\text { Comportamento observado no } \\
\text { animal }\end{array}$ & Descrição \\
\hline Ajoelhar & Animal se ajoelha durante a condução dentro do tronco de contenção ou brete \\
\hline Deitar & Animal se deita durante a condução dentro do tronco de contenção ou brete \\
\hline $\begin{array}{l}\text { Subir sobre outro animal } \\
\text { subsequente }\end{array}$ & Animal sobe sobre outro animal subsequente \\
\hline Tentar fugir/pular do brete & Animal tenta fugir do tronco, tenta pular para fora do brete \\
\hline Reagir à introdução da agulha & $\begin{array}{c}\text { Animal reage à introdução da agulha: pula, dá um arranque, movimenta a cabeça } \\
\text { e pescoço }\end{array}$ \\
\hline Deslocamento & Animal se desloca no momento da aplicação \\
\hline Vocalização & Animal vocaliza em algum momento durante a condução no tronco ou brete \\
\hline
\end{tabular}

Tabela 2 Ações do operador durante o processo de vacinação e da ocorrência de reações clínicas nos animais após a aplicação da vacina.

\begin{tabular}{cc}
\hline $\begin{array}{c}\text { Ações do operador e reações após a } \\
\text { aplicação }\end{array}$ & Descrição \\
\hline Introdução repetitiva da agulha & $\begin{array}{r}\text { Número de vezes que o operador teve que proferir a introdução da agulha } \\
\text { no animal para finalizar uma única aplicação }\end{array}$ \\
Sangramento no local da aplicação & $\begin{array}{r}\text { Ocorrência de sangramentos visíveis logo após a aplicação da vacina } \\
\text { Aplicação da vacina em outro local que não seja no teço médio do } \\
\text { pescoço }\end{array}$ \\
Aplicação da vacina em local incorreto & Vocalização negativa do operador frente às ações do animal \\
Perda de dose de vacina & Perdas de dose de vacina devido a erros na aplicação \\
Tempo de execução do trabalho & Tempo em segundos observado entre o início e final do manejo \\
\hline
\end{tabular}

As observações eram iniciadas a partir do momento em que os animais começavam a entrar no brete coletivo, e finalizadas no momento em que os mesmos saíam do tronco de contenção. Para os procedimentos de colheita de dados, quatro observadores treinados registraram os comportamentos conforme o método de observação contínua focal, descrito por Martin e Bateson (1993), sendo cada observador considerado como uma repetição. As análises de variância foram realizadas através do procedimento General Linear Model (GLM) do programa SAS $^{\circledR} \quad$ (SAS Institute2002), com diferenças entre os tratamentos RAC e CON analisados pelo Teste - T, bem como pelo método qui- 
quadrado, quando conveniente.

\section{Resultados}

Os animais analisados neste estudo, independente da categoria, apresentaram comportamentos de caráter menos aversivo quando submetidos ao manejo racional durante a vacinação (tabelas 3 e 4).

$\mathrm{Na}$ categoria de fêmeas adultas, foram observadas diferenças estatísticas nos percentuais de reações como deslocamento $(\mathrm{P}<0,001)$ e reação à introdução da agulha $(\mathrm{P}<0,01)$, além dos indicadores de eficiência denominados perda de dose de vacina $(\mathrm{P}<0,05)$, sangramento no local da aplicação, tempo de execução do trabalho e aplicação da vacina em local incorreto $(\mathrm{P}<0,001)$.

Em relação aos machos jovens, foram observados valores distintos apenas com relação ao tempo de execução do trabalho e aplicação da vacina em local incorreto $(\mathrm{P}<0,001)$, além de diferenças nos percentuais de comportamentos como deslocamento $(\mathrm{P}<0,01)$ e tentativa de fugir/pular do brete $(\mathrm{P}<0,05)$.

No caso da categoria de bezerros nota-se que os indicadores de eficiência como introdução repetitiva da agulha $(\mathrm{P}<0,05)$, sangramento no local da aplicação $(\mathrm{P}<0,05)$, tempo de execução do trabalho $(\mathrm{P}<0,001)$ e percentuais de comportamentos como deslocamento $(\mathrm{P}<0,001)$, reagir à introdução da agulha $(\mathrm{P}<0,01)$, subir sobre outro animal e tentar fugir/pular do brete $(\mathrm{P}<0,05)$ também diferiram entre os dois tipos de manejo aplicados. Evidencia-se, assim como nas demais categorias, uma redução na reatividade e na perda de eficiência de trabalho quando manejados de forma racional.

Tabela 3 Média de ocorrências de reações durante o processo de vacinação executado sob manejo Racional (RAC) e Convencional (CON) nas diferentes categorias.

\begin{tabular}{|c|c|c|c|c|c|c|}
\hline \multirow{2}{*}{ Comportamento } & \multicolumn{2}{|c|}{ Fêmeas Adultas } & \multicolumn{2}{|c|}{ Machos Jovens } & \multicolumn{2}{|c|}{ Bezerros } \\
\hline & RAC & $\mathrm{CON}$ & RAC & $\mathrm{CON}$ & RAC & $\mathrm{CON}$ \\
\hline Ajoelhar (\%) & 5,1 & 3,0 & 5,0 & 10,3 & 7,2 & 4,2 \\
\hline Deitar $(\%)$ & 5,1 & 3,0 & 10,0 & 19,0 & 14,4 & 24,2 \\
\hline Subir sobre outro animal (\%) & 0,7 & 0,8 & 0,0 & 3,4 & 0,0 & $5,8^{*}$ \\
\hline Tentar fugir/pular do brete (\%) & 0,0 & 1,5 & 0,0 & $6,9^{*}$ & 1,0 & $7,5^{*}$ \\
\hline $\begin{array}{c}\text { Reagir à introdução da } \\
\text { agulha }(\%)\end{array}$ & 12,5 & $25,0 * *$ & 15,0 & 15,5 & 14,4 & $29,2^{* *}$ \\
\hline Deslocamento (\%) & 2,2 & $24,2 * * *$ & 0,0 & $15,5^{* *}$ & 6,2 & $32,5 * * *$ \\
\hline Vocalização (\%) & 2,2 & 0,8 & 3,3 & 1,7 & 7,2 & 12,5 \\
\hline
\end{tabular}

Médias na mesma linha acompanhadas de * diferem significativamente entre si dentro de cada categoria (fêmeas adultas; machos jovens; bezerros): $*=\mathrm{P}<0,05 ; * *=\mathrm{P}<0,01 ; * * *=\mathrm{P}<0,001$.

\section{Discussão}

As diferenças entre os manejos observadas nos indicadores como vocalização do operador, introdução repetitiva da agulha no animal, sangramento no local da aplicação, perda de vacina, aplicação da vacina em local incorreto, tempo de execução do trabalho, reatividade e deslocamento, no caso das fêmeas adultas, podem ser explicadas pelo fato de que neste tipo de manejo a maior reatividade do animal e deslocamento do mesmo acarreta dificuldades na contenção, explicando maiores perdas nas doses de vacina, sangramento devido à má aplicação e aumento no tempo de serviço. Da mesma forma, a dificuldade em conter o animal gera certo receio na aproximação, fazendo com que aplicação da vacina em local incorreto seja feita, muitas vezes por medo devido ao tamanho do animal e a presença de chifres, considerando que são animais já adultos.

Diversas pesquisas apontam o estresse de manejo como o principal precursor de comportamentos aversivos, considerando que os bovinos são animais capazes de discriminar pessoas envolvidas nas atividades. Tal afirmação evidencia a importância de estabelecermos uma rotina de manejo adequada, pois, conforme Voisinet et al (1997), animais mais reativos apresentam menores ganhos de peso, afetando seu desempenho. Além disso, diversos aspectos da 
criação podem ser afetados caso a fêmea não esteja em condições propícias para expressar seu potencial produtivo, como por exemplo a reprodução, afetada por meio da ação de hormônios que interferem na maturação folicular, verificando-se problemas como ausência de cio, abortos e/ou reabsorção embrionária e, consequentemente, aumento no período de intervalo entre partos do rebanho, diminuição na produção de bezerros da propriedade, menor produção de leite devido aos distúrbios fisiológicos ou até mesmo por lesões causadas no úbere durante uma condução e/ou manejo inadequado (Breen et al 2005; Etim et al 2013).

Tabela 4 Média de ocorrências de indicadores de eficiência dos manejos Racional (RAC) e Convencional (CON) nas diferentes categorias durante o processo de vacinação

\begin{tabular}{|c|c|c|c|c|c|c|}
\hline \multirow{2}{*}{ Comportamento } & \multicolumn{2}{|c|}{ Fêmeas Adultas } & \multicolumn{2}{|c|}{ Machos Jovens } & \multicolumn{2}{|c|}{ Bezerros } \\
\hline & RAC & $\mathrm{CON}$ & RAC & $\mathrm{CON}$ & RAC & $\mathrm{CON}$ \\
\hline $\begin{array}{c}\text { Introdução repetitiva da } \\
\text { agulha }(\%)\end{array}$ & 0,7 & 3,0 & 1,7 & 1,7 & 0,0 & $4,2 *$ \\
\hline $\begin{array}{l}\text { Sangramento no local da } \\
\text { aplicação (\%) }\end{array}$ & 11,4 & $52,8 * * *$ & 8,3 & 15,8 & 10,3 & $20,8^{*}$ \\
\hline $\begin{array}{c}\text { Aplicação da vacina em local } \\
\text { incorreto }(\%)\end{array}$ & 0,0 & $100,0 * * *$ & 0,0 & $100,0 * * *$ & 0,0 & 0,0 \\
\hline Vocalização do operador (\%) & $9,6^{*}$ & 3,0 & 0,0 & 1,7 & 4,1 & 5,8 \\
\hline Perda de dose de vacina $(\%)$ & 0,0 & $3,8^{*}$ & 0,0 & 1,7 & 1,0 & 4,2 \\
\hline $\begin{array}{l}\text { Tempo de execução do } \\
\text { trabalho (segundos) }\end{array}$ & 47,1 & $54,3^{* * *}$ & 54,8 & $62,2 * * *$ & 53,9 & $68,1 * * *$ \\
\hline
\end{tabular}

Médias na mesma linha acompanhadas de * diferem significativamente entre si dentro de cada categoria (fêmeas adultas; machos jovens; bezerros): * $=\mathrm{P}<0,05 ; * *=\mathrm{P}<0,01 ; * * *=\mathrm{P}<0,001$.

Nos machos jovens, o dispêndio maior de tempo na execução do trabalho observado no manejo racional ocorreu em função da necessidade de contenção do animal no tronco para identificação. Já a aplicação da vacina em local incorreto, assim como nas fêmeas adultas, pode estar relacionada ao fato de que estes animais já apresentam chifres, o que causa receio no funcionário no momento da aplicação, fazendo com que o mesmo, muitas vezes, prefira aplicar em outro local para evitar uma aproximação. Tal observação se torna de extrema importância quando se avalia carcaças de bovinos em relação a perdas por abcessos e hematomas. Em estudo envolvendo 13000 carcaças observou-se uma maior frequência de abscessos (36,3\%) em relação aos hematomas $(11,4 \%)$. Dos hematomas encontrados no traseiro, os cortes mais atingidos foram: costela do traseiro $(37,99 \%)$, coração da alcatra $(21,20 \%)$, picanha $(17,15 \%)$ e lagarto $(22,05 \%)$, além dos demais cortes que juntos somaram 1,61\%. Já dos abscessos que acometeram as carcaças, os cortes mais atingidos foram: cupim $(80,12 \%)$, acém $(15,6 \%)$, costela do dianteiro $(3,2 \%)$, e todos os outros cortes que juntos somaram 1,08\% (Rezende-Lago, D' Amato e Marchi 2011). Estes dados ressaltam a importância na escolha correta dos locais de aplicação dos produtos e de um manejo adequado, evitando assim, hematomas ou lesões medicamentosas em cortes cárneos nobres do animal em porcentagens relativamente elevadas.
Além disso, as diferenças observadas nos percentuais de machos jovens apresentando comportamentos como deslocamento e tentativa de fugir/pular do brete, os quais ocorreram com maior intensidade no manejo convencional, demonstram uma possível sensação de ameaça do mesmo para com o manejo empregado. Algumas ações permitem que os animais sejam manejados com tranquilidade e ainda associem o ambiente a um lugar seguro, como por exemplo, permitir que os mesmos ao serem conduzidos a andar por um curral e tronco novos, possam investigar com calma o local ou até mesmo fornecer sua comida favorita durante os procedimentos, de forma a criar uma associação positiva com o curral. Entretanto, se os animais passarem por procedimentos assustadores ou de dor, é provável que ocorra uma associação negativa em relação ao local, dificultando manejos futuros (Grandin 2008).

No caso dos bezerros, em relação ao tempo de execução do trabalho, maiores médias foram obtidas durante o manejo convencional, o que provavelmente também ocorreu em função da necessidade de contenção do animal no tronco para sua identificação. Este tipo de manejo também apresentou uma maior frequência de sangramento devido à falta de controle durante a aplicação, acarretando um impacto maior da pistola em contato com o couro do animal. $\mathrm{O}$ fato da média de aplicação da vacina em local incorreto não ter se mostrado significativa pode estar relacionado à idade dos animais que, por serem jovens ainda não possuem chifres, como no caso dos animais jovens ou das fêmeas adultas. 
Durante o manejo desta categoria, melhorias significativas através de ações do operador puderam ser observadas no manejo racional, favorecendo a interação entre o aplicador e animal, além de proporcionar um melhor resultado em relação à ocorrência de contusões e/ou lesões decorrentes da má aplicação. Apesar da ocorrência semelhante de comportamentos como deitar, ajoelhar e vocalizar nos dois tipos de manejo, os demais comportamentos estudados foram menores durante o manejo racional. Em estudo com bezerros leiteiros o manejo gentil, além de melhorar os índices produtivos, reduziu a tendência dos animais a evitarem o homem, aumentando a capacidade de uma aproximação segura, o que indica a importância de interações positivas para o aumento do bem-estar e redução dos riscos de acidentes (Lensink, Veissier e Florand 2000).

Ao compararmos os dados referentes aos diferentes métodos executados, observa-se uma relação mais harmônica quando se aplica o manejo racional, ilustrada por menores médias obtidas em relação à reatividade dos bovinos, com redução na porcentagem de comportamentos aversivos e perdas de material durante o manejo de vacinação. Animais mais excitáveis ou agitados podem ser problemáticos quanto ao manejo durante sua comercialização, visto que representam riscos à integridade física dos outros animais, dos trabalhadores e dos seres humanos de forma geral (Lanier et al 2001). Além disso, seu desempenho produtivo futuro pode ser prejudicado com lesões na carcaça e queda no desempenho, o que não é desejável quando se trata de animais destinados à produção de carne.

\section{Conclusões}

O manejo racional diminui possíveis interações negativas que conduzam a reações de estresse e mudanças comportamentais que possam afetar o desempenho do rebanho, além de facilitar a execução do manejo, proporcionando não só um aumento na eficiência em relação ao serviço, mas também uma melhor interação homem/animal, o que, consequentemente, contribuirá para a obtenção de índices produtivos satisfatórios, tendo como resultado um produto final de melhor qualidade.

\section{Referências}

Breen KM, Billings HJ, Wagenmaker ER, Wessinger EW, Karsch FJ (2005) Endocrine basis for disruptive effect of cortisol on preovulatory events. Endocrinology 146: 2107-2115.

Etim NN, Offiong EEA, Udo MD, Williams ME, Evans EI (2013) Physiological Relationship between Stress and Reproductive Efficiency. Agriculture and Biology Journal of North America 4: 600-604.

Grandin T (2008) Human e livestock handling. $1^{\circ}$.Storey Pub., Massachusetts.
Lanier JL, Grandin T, Green R, Avery D, Mcgee K (2001) A note on hair whorl position and cattle temperament in the auction ring. Applied Animal Behaviour Science 73: 93-101.

Lensink BJ, Veissier I, Florand L (2000) The farmer's influence on calves'behaviour, health and production of a veal unit. Animal Science 72: 105-116.

Martin P, Batenson P (1993) Measuring behaviour: an introductory guide. Cambridge University Press, Cambridge.

Rezende-Lago NCM, D’Amato CC, Marchi PGF (2011) Perdas econômicas por abcessos e hematomas em carcaças de bovinos. Revista Eletrônica Univar 2: 174-177.

Pajor EA, Rushen J, De Passiélle AM (2000) Aversion learning techniques to evaluate dairy cattle handling practices. Applied Animal Behaviour Science 69: 89-102.

Pinheiro AA, Brito IF (2009) Bem-estar e Produção Animal. http://www.infoteca.cnptia.embrapa.br/bitstream/doc/748310/1/doc 93.pdf. Acessado em: 14 fevereiro de 2015.

SAS (2002) User's guide: statistics. Cary: SAS Institute Inc.

Voisinet BD, Grandin T, O'Connor SF, Tatum JD, Deesing MJ (1997) Bos indicus-cross feedlot cattle with excitable temperaments have tougher meat and a higher incidence of borderline dark cutters. Meat Science 46: 367-377. 